\title{
Multiuser Diversity with Capture for Wireless Networks: Protocol and Performance Analysis
}

\author{
Justin Foo and Defeng (David) Huang, Senior Member, IEEE
}

\begin{abstract}
In a wireless network, with the aid of rate adaptation, multiuser diversity can be exploited by allowing the mobile user with the best channel to use the channel. However, the overhead that results from polling mobile stations to obtain channel state information (CSI) in large networks can outweigh the multiuser diversity gain. In this paper, we propose a wireless medium access control protocol, namely Multiuser Diversity with Capture (MDC), which explicitly employs the capture effect to obviate the overhead problem. We analyze the goodput performance of MDC and compare it with the Medium Access Diversity (MAD) scheme proposed in the literature. Our results show that MDC is effective in networks with radio receivers possessing reasonably good capture properties and in networks where the number of mobile stations is reasonably large.
\end{abstract}

Index Terms - capture effect, MAC protocol, multiuser diversity, wireless LAN

\section{INTRODUCTION}

M ULTIUSER diversity is the inherent diversity in channel quality exhibited in a time-varying wireless channel across different mobile users at different times. The earliest work on multiuser diversity was performed by Knopp and Humblet [1], who demonstrated that the capacity of the uplink channel of a wireless network is maximized by only allowing the user with the best channel to transmit at any given time. Multiuser diversity has been investigated in slotted ALOHA systems [2] and in the downlink scheduling for Code Division Multiple Access (CDMA) cellular networks, such as the High Data Rate (HDR) system [3] and the High-Speed Downlink Packet Access (HSDPA) system [4] for wideband CDMA.

Conventionally, when multiuser diversity is exploited in a centralized wireless network, channel state information (CSI) must be obtained at the base station (BS), thereby inducing huge system overhead. This overhead can significantly decrease system goodput when there is a large number of mobile stations (MSs) in the network. In [5], the system overhead issue is mitigated through the use of a proposed user identification scheme, where CSI from multiple MSs is obtained at the base station simultaneously. In [6], signal to

Manuscript received November 1, 2007; revised April 15. This research was supported under Australian Research Council's Discovery Projects funding scheme (project number DP0877616). This work was presented in part at the IEEE Wireless Communications and Networking Conference 2007 in Hong Kong and the IEEE International Conference on Communications 2008 in Beijing. This paper is dedicated to Dr. Guven Mercankosk, who passed away after his brave fight against liver cancer on January 4, 2007.

Justin Foo is with the Western Australia Telecommunications Research Institute and the School of Electrical, Electronic and Computer Engineering at the University of Western Australia (e-mail: fooj@watri.org.au).

Defeng (David) Huang is with the School of Electrical, Electronic and Computer Engineering at the University of Western Australia (email: huangdf@ee.uwa.edu.au).

Digital Object Identifier 10.1109/JSAC.2008.081005. noise ratio (SNR) thresholding was proposed to reduce the amount of bandwidth required for CSI feedback on dedicated or contention-based uplink channels. In [7], Medium Access Diversity (MAD) was proposed where a small subset of the MSs in the network are polled each time for CSI, thereby reducing the system overhead. However, in polling the CSI of only a small subset of MSs, there is a potential loss of goodput since the MS with the best channel may not be in the chosen subset. Nonetheless, with packet concatenation and revenuebased scheduling, the results in [7] based on the free space path loss and Rayleigh fading propagation models showed a significant goodput improvement over conventional rate adaptation techniques such as Auto Rate Fallback (ARF) [8] and Opportunistic Auto Rate (OAR) [9].

In wireless communications, the capture effect is the ability of a radio receiver to receive a signal from one transmitter in the presence of interference from one or more other transmitters. By employing the capture effect, theoretical analysis of wireless slotted ALOHA networks has demonstrated that significantly higher throughput can be achieved compared with the case that capture is not considered [10]-[13]. In [14], Kim and Lee analyzed the effects of capture on carrier sense multiple access/collision avoidance (CSMA/CA) protocols, demonstrating that the network throughput is sensitive to the capture ratio, multipath fading and shadowing effects. In a recent investigation [15], the capture effect was exploited to reduce system overhead and achieve multiuser diversity gain for reservation slotted ALOHA and reservation slotted nonpersistent inhibit sense multiple access (ISMA) systems.

In this paper, we present a new wireless medium access control (WMAC) protocol called Multiuser Diversity with Capture (MDC) to exploit multiuser diversity. In MDC, the capture effect is utilized to reduce the overhead in a wireless network with one BS and multiple MSs. The explicit use of the capture effect is motivated by the fact that prior to data transmission, the BS only needs to find the MS with the best channel and its CSI. In the proposed MDC, we allow multiple MSs simultaneously to transmit signals to the BS. The capture effect is then exploited at the BS to identify the MS with the strongest signal and therefore the best channel. In contrast to conventional CSI feedback reduction schemes [6], [16], where more than one MS's CSI is transmitted to the $\mathrm{BS}$, in MDC, only one MS's CSI is fed back to the BS in each transmission cycle, thereby reducing the overhead. We develop a general analytical framework for determining the expected goodput of the MDC protocol. Using this framework, we derive a closed-form solution for the expected goodput for MDC over Rayleigh fading channels. Our numerical results 


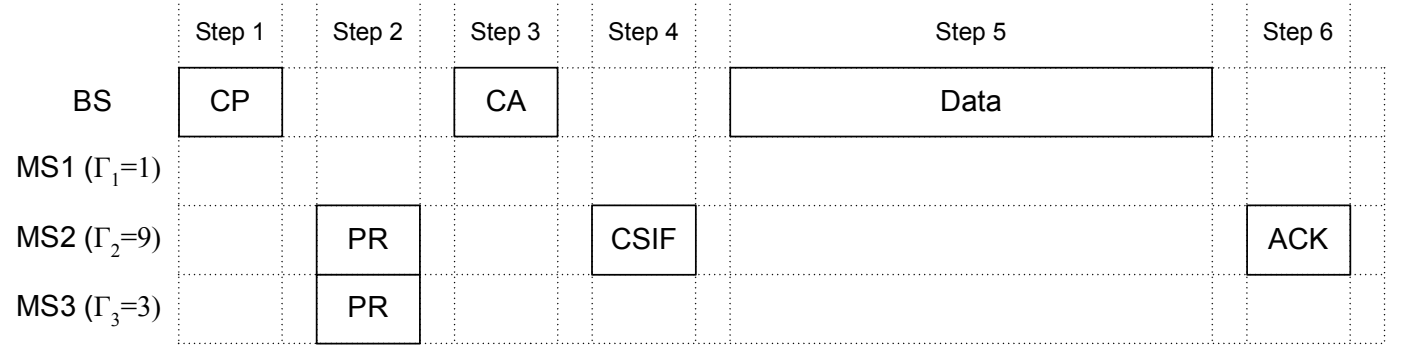

Fig. 1. Example MDC transmission cycle for $\gamma=2$ and $z=2.5$.

for Rayleigh fading channels show that by explicitly using the capture effect to reduce system overhead, MDC achieves much better goodput performance than MAD, especially when the radio receiver has reasonably good capture properties or when the number of MSs is reasonably large. We then consider an application of MDC based on the IEEE 802.11a system [17] under a wireless environment with path loss, shadowing and Rayleigh fading. The simulation results manifest the same trends as in the numerical results for Rayleigh fading channels.

This paper is organized as follows. Section II introduces the MDC protocol and the mathematical model for the capture effect. The analysis of the goodput of the MDC protocol is presented in Section III. In Section IV, we describe an application of MDC based on the IEEE 802.11a system. Simulation results are presented in V. Finally, Section VI concludes this paper.

\section{MDC PROTOCOL}

\section{A. System Description}

We consider a wireless network with one BS and $N$ MSs, indexed from 1 to $N$. We assume that a constant transmission power $P_{T}$ is used for all packet transmissions and that the uplink and downlink channels are reciprocal. We also assume that whenever exactly one MS in the network transmits a packet, that packet is always received successfully. Channel quality is measured by the instantaneous signal to noise ratio (SNR) between an MS and the BS and we define this to be the channel state of the MS. The total duration required to transmit a data packet, including the transmission time of the control packets that setup the data transmission, is assumed to be less than the channel coherence time. Consequently, the channel state of an MS is constant over the total duration required to transmit a data packet. We use $\Gamma_{i}$ to denote the $i$ th MS's channel state. For simplicity, we further assume that channel estimation is perfect. Thus, we will not differentiate between $\Gamma_{i}$ and its estimate.

In the following, we describe the MDC protocol for downlink data transmission, where every data packet transmission takes place as part of a transmission cycle. Each transmission cycle of the MDC protocol consists of the following six steps:

\section{Step 1. Channel Probing}

At the start of the transmission cycle, the BS broadcasts a channel probe $(\mathrm{CP})$ packet, prompting the MSs to respond.

\section{Step 2. Channel Competition}

Each MS uses the CP packet to estimate its channel state.
For MS $i$, if the SNR $\Gamma_{i}>\gamma$, then MS $i$ responds to the BS with a probe response (PR) packet ${ }^{1}$. The system parameter $\gamma$ is called the response threshold.

\section{Step 3. Channel Allocation}

Based on the received PR packets, the BS seeks to identify the MS with the best channel and allocate it the rest of the transmission cycle so that it may perform data transmission. However, the BS will only be able to correctly identify the MS with the best channel if (i) there is exactly one responding MS, or (ii) the strongest PR signal can be captured from multiple responding MSs. When the MS with the best channel is identified, that MS is allocated the rest of the transmission cycle. Otherwise, the BS has not gained any information about which MS has the best channel and it allocates the rest of the transmission cycle to a randomly chosen MS. By randomly chosen, we mean that the index of the MS is a uniformly distributed random variable from 1 to $N$. When the BS has decided which MS will be allocated with the rest of the transmission cycle, it then sends a channel allocation (CA) packet. The selected MS is called the winning $M S$, which we will index by $w$.

\section{Step 4. CSI Feedback}

The winning MS, MS $w$, sends a channel state information feedback (CSIF) packet to the BS so that the exact CSI can be obtained by the BS.

\section{Step 5. Data Packet Transmission}

Based on the channel state estimate $\Gamma_{w}$, the most suitable data rate is selected by the BS and a data packet is transmitted from the BS to MS $w$.

\section{Step 6. Data Packet Acknowledgement}

Upon the successful reception of the data packet, MS $w$ replies with an acknowledgement (ACK) packet.

Fig. 1 depicts an example of the operation of MDC where there are three MSs. In the first step, the BS broadcasts a CP packet to all MSs. In the second step, MSs 2 and 3 will both reply with a PR packet and MS 1 will not reply since $\Gamma_{1}<\gamma$. In the third step, the BS is able to capture the PR packet from

\footnotetext{
${ }^{1}$ In this paper, we assume that the PR packets do not explicitly carry CSI. As a result, only the identity of the best MS is required to be extracted by the BS. If CSI is explicitly carried in the PR packets, Steps 3 and 4 are not required when the best MS is captured by the BS at the channel competition step. However, when no MS is captured by the BS, Steps 3 and 4 are still needed. Furthermore, when CSI is explicitly carried, a good receiver is required to capture and decode the PR packet of the best MS to extract both its identity and its CSI. Nonetheless, our analysis in Section III can still be applied in this scenario, by proper redefinition of the rate adaptation function $g(x)$ for the cases where Steps 3 and 4 are used and not used.
} 
MS 2 since $\Gamma_{2}>z \Gamma_{3}$, where $z$ is the capture ratio and will be described in detail in Section II-B. In the fourth step, MS 2 sends a CSIF packet to the BS, which allows the BS to obtain the channel state of MS 2. In the fifth step, the BS selects a suitable data rate based on the channel state of MS 2 and transmits the data packet. Finally, in the sixth step, MS 2 transmits an ACK packet to indicate successful reception of the data packet.

For uplink data transmission, the operation of the MDC protocol is similar, but the CSIF packet is not needed. After MS $w$ receives the CA packet in Step 3, it has an estimate of its own channel state $\Gamma_{w}$. Consequently, MS $w$ can immediately select the most suitable data rate and transmit a data packet directly to the BS. In the final step of the uplink data transmission cycle, when the BS successfully receives the data packet, it replies to the winner MS $w$ with an ACK packet. Since the CSIF packet is not needed in uplink data transmission, less overhead is required than in downlink data transmission and higher goodput is manifested. In this paper, our focus is on downlink data transmission.

\section{B. Capture Model}

In the context of collision-based random access networks, the capture effect refers to the fact that a radio receiver may successfully decode a radio signal from one user despite the presence of interference from other users. It is an inherently complex phenomenon that depends on the specific implementation of the receiver. Several mathematical models have been proposed in the literature to characterize the capture effect [18], [19]. In this paper, we adopt the capture ratio model, which is the predominant model for the capture effect in the literature. According to this model, a signal is considered to be captured (i.e., decoded correctly by the receiver) when the ratio of the received signal power to the total interference power is greater than a threshold factor, known as the capture ratio.

In terms of the MDC protocol, let $n>1$ be the number of responding MSs. Let the indices of the responding MSs be $v_{1}, v_{2}, \ldots, v_{n}$. Without loss of generality, let $v_{1}$ be the index of the MS with the strongest signal. Then the PR packet from MS $v_{1}$ will be captured if and only if:

$$
\Gamma_{v_{1}}>z \sum_{i=2}^{n} \Gamma_{v_{i}}
$$

where $z$ is the capture ratio. In this paper, we assume that $z \geq 1$.

\section{Goodput ANALYSIS}

\section{A. Goodput Analysis of MDC over General Channels}

Let $R_{n}$ denote the event that $n$ of the $N$ MSs in the network send a PR packet and let $G$ be the random variable that represents the goodput of the MDC system. Applying the law of total expectation, the expected goodput of MDC is

$$
E[G]=\sum_{n=0}^{N} E\left[G \mid R_{n}\right] P\left(R_{n}\right)
$$

Let $C$ denote the event that the BS is able to correctly identify the MS with the best channel during the channel competition step. A second application of the law of total expectation yields:

$$
E\left[G \mid R_{n}\right]=E\left[G \mid C, R_{n}\right] P\left(C \mid R_{n}\right)+E\left[G \mid \bar{C}, R_{n}\right] P\left(\bar{C} \mid R_{n}\right)
$$

where we use the bar notation $\bar{C}$ to represent the set complement of $C$.

To derive the expressions for $P\left(R_{n}\right), P\left(C \mid R_{n}\right), P\left(\bar{C} \mid R_{n}\right)$, $E\left[G \mid C, R_{n}\right]$ and $E\left[G \mid \bar{C}, R_{n}\right]$ in (2) and (3), we make the following assumptions and definitions. We assume that the MSs experience fading independently, hence the $\Gamma_{i}$ s are independent and identically distributed (i.i.d.) random variables, each with probability density function (pdf) $f_{\Gamma}(\cdot)$ and cumulative distribution function (cdf) $F_{\Gamma}(\cdot)$.

For the channel competition step, let the indices of the MSs that do not send a PR packet to the BS be $u_{1}, u_{2}, \ldots, u_{N-n}$ and note that the indices of the MSs that do send PR packets to the $\mathrm{BS}$ are $v_{1}, v_{2}, \ldots, v_{n}$. Since the $\Gamma_{i} \mathrm{~s}$ are i.i.d., it follows that the $\Gamma_{u_{j}}$ s are i.i.d. and that the $\Gamma_{v_{k}}$ s are also i.i.d.. Denote the pdfs of $\Gamma_{u_{j}}$ and $\Gamma_{v_{k}}$ by $f_{U}(\cdot)$ and $f_{V}(\cdot)$, and their cdfs by $F_{U}(\cdot)$ and $F_{V}(\cdot)$, respectively. It is easily shown that

$$
\begin{gathered}
f_{U}(x)=\frac{f_{\Gamma}(x)}{F_{\Gamma}(\gamma)}, \quad 0 \leq x<\gamma, \\
F_{U}(x)=\frac{F_{\Gamma}(x)}{F_{\Gamma}(\gamma)}, \quad 0 \leq x<\gamma, \\
f_{V}(x)=\frac{f_{\Gamma}(x)}{1-F_{\Gamma}(\gamma)}, \quad x \geq \gamma, \\
F_{V}(x)=\frac{F_{\Gamma}(x)-F_{\Gamma}(\gamma)}{1-F_{\Gamma}(\gamma)}, \quad x \geq \gamma .
\end{gathered}
$$

Further, let the pdf and cdf of the sum of $n$ independent random variables with pdf $f_{V}(\cdot)$ be $f_{V, n}(\cdot)$ and $F_{V, n}(\cdot)$, respectively. It follows that $f_{V, n}(\cdot)$ is the $n$-fold convolution of $f_{V}(\cdot)$. For convenience, we define $f_{V, 0}(t)=\delta(t)$, where $\delta(\cdot)$ is the Dirac delta function. Let $g(x)$ be the transmitted goodput in a transmission cycle for a winning MS with a given channel state $x$.

As demonstrated in the Appendix,

$$
P\left(R_{n}\right)=\left(\begin{array}{c}
N \\
n
\end{array}\right)\left(1-F_{\Gamma}(\gamma)\right)^{n} F_{\Gamma}(\gamma)^{N-n},
$$

$P\left(C \mid R_{n}\right)= \begin{cases}0, & n=0 \\ 1, & n=1 \\ n \int_{(n-1) \gamma}^{\infty}\left(1-F_{V}(z x)\right) f_{V, n-1}(x) d x, & n \geq 2\end{cases}$

$$
P\left(\bar{C} \mid R_{n}\right)=1-P\left(C \mid R_{n}\right)
$$

$$
E\left[G \mid C, R_{n}\right]= \begin{cases}0, & n=0 \\ \int_{\gamma}^{\infty} g(x) f_{V}(x) d x, & n=1 \\ \frac{n}{P\left(C \mid R_{n}\right)} a_{n}, & n \geq 2\end{cases}
$$


where

$$
a_{n}=\int_{z(n-1) \gamma}^{\infty} g(x) F_{V, n-1}\left(\frac{x}{z}\right) f_{V}(x) d x
$$

and

$$
E\left[G \mid \bar{C}, R_{n}\right]= \begin{cases}\int_{0}^{\gamma} g(x) f_{U}(x) d x, & n=0 \\ 0, & n=1 \\ \frac{N-n}{N} E\left[G \mid \bar{C}, R_{0}\right]+\frac{n}{N P\left(\bar{C} \mid R_{n}\right)} b_{n}, & n \geq 2\end{cases}
$$

where

$$
b_{n}=E\left[G \mid C, R_{1}\right]-a_{n}-(n-1) c_{n},
$$

and

$c_{n}=\int_{\gamma}^{\infty} \int_{(n-2) \gamma}^{\infty} g(x) f_{V}(x)\left(1-F_{V}(z(x+y))\right) f_{V, n-2}(y) d y d x$.

From (2), (3) and (8)-(15), it can be seen that the expected goodput for MDC is completely described by:

- the number of MSs in the network, $N$;

- the capture ratio, $z$;

- the response threshold, $\gamma$;

- the pdf of the SNR of a received signal, $f_{\Gamma}(\cdot)$, which is determined by the wireless channel and the transmit power $P_{T}$; and

- the rate adaptation function, represented by $g(\cdot)$.

In general, $E[G]$ is not closed form because it is usually not possible to derive a closed form expression for $f_{V, n}(\cdot)$. In the next section, we analyze the Rayleigh fading channel, where $f_{V, n}(\cdot)$ has a closed form expression.

\section{B. Goodput Analysis of MDC over Rayleigh Fading Channels}

For Rayleigh fading channels, the received signal amplitudes at the BS are Rayleigh distributed, thus $\Gamma_{i}$ is exponentially distributed for all $i$. Hence,

$$
f_{\Gamma}(x)=\mu e^{-\mu x}, \quad x>0
$$

and

$$
F_{\Gamma}(x)=1-e^{-\mu x}, \quad x>0
$$

where $\mu$ is a constant that is inversely proportional to the transmit power $P_{T}$.

We assume that the physical layer (PHY) is capable of rate adaptation and selects the highest feasible data rate given the channel state of the winning MS. Specifically,

$$
g(x)= \begin{cases}0, & x<m_{1} \\ g_{i}, & m_{i} \leq x<m_{i+1}, \forall i<M \\ g_{M}, & x \geq m_{M}\end{cases}
$$

where for $1 \leq i \leq M, g_{i}$ is the amount of payload transmitted per unit time over the transmission cycle for PHY mode $i, M$ is the number of PHY modes and $m_{i}$ is the minimum SNR required to transmit at PHY mode $i$. We will refer to $m_{i}$ as the mode threshold for PHY mode $i$.

By substituting (17) into (8), we have

$$
P\left(R_{n}\right)=\left(\begin{array}{c}
N \\
n
\end{array}\right) e^{-n \mu \gamma}\left(1-e^{-\mu \gamma}\right)^{N-n} .
$$

To find the expressions for $P\left(C \mid R_{n}\right), E\left[G \mid C, R_{n}\right]$ and $E\left[G \mid \bar{C}, R_{n}\right]$, the computation of $f_{V, n}(\cdot)$ is required. Using (16) and (6), it can be shown by induction that

$$
f_{V, n}(x)=\frac{(\mu(x-n \gamma))^{n-1}}{(n-1) !} \mu e^{-\mu(x-n \gamma)} .
$$

By putting (17), (7) and (20) into (9), it can be shown after some algebraic manipulations that for $n \geq 2$,

$$
P\left(C \mid R_{n}\right)=\frac{n e^{-\mu \gamma((n-1) z-1)}}{(z+1)^{n-1}} .
$$

To obtain $E\left[G \mid C, R_{n}\right]$ and $E\left[G \mid \bar{C}, R_{n}\right]$, it is necessary to compute integrals involving $g(\cdot)$. Define $m_{0}=-\infty, m_{M+1}=$ $\infty$ and the function

$$
\zeta(x)=\underset{1 \leq i \leq M}{\operatorname{argmax}}\left\{m_{i} \mid m_{i} \leq x\right\} .
$$

Then, for any function $h(\cdot)$ with antiderivative $H(\cdot)$, we have (using (18))

$$
\begin{aligned}
\int_{a}^{b} g(x) h(x) d x= & g_{\zeta(a)} \cdot\left(H\left(m_{\zeta(a)+1}\right)-H(a)\right) \\
& +g_{\zeta(b)} \cdot\left(H(b)-H\left(m_{\zeta(b)}\right)\right) \\
& +\sum_{i=\zeta(a)+1}^{\zeta(b)-1} g_{i} \cdot\left(H\left(m_{i+1}\right)-H\left(m_{i}\right)\right)
\end{aligned}
$$

Using (23), we can directly compute $E\left[G \mid \bar{C}, R_{0}\right]$ with $H(\cdot)$ replaced by $F_{U}(\cdot)$ and $E\left[G \mid C, R_{1}\right]$ with $H(\cdot)$ replaced by $F_{V}(\cdot)$, respectively. To compute $E\left[G \mid \bar{C}, R_{n}\right]$ and $E\left[G \mid C, R_{n}\right]$ for $n \geq 2$, it suffices to compute $a_{n}$ and $c_{n}$. For $n \geq 2$, define

$$
\alpha_{n}(x)=\int f_{V}(x) F_{V, n-1}\left(\frac{x}{z}\right) d x
$$

and

$\beta_{n}(x)=\iint_{(n-2) \gamma}^{\infty} f_{V}(x)\left(1-F_{V}(z(x+y))\right) f_{V, n-2}(y) d y d x$

so that

$$
a_{n}=\int_{z(n-1) \gamma}^{\infty} g(x) d \alpha_{n}(x)
$$

and

$$
c_{n}=\int_{\gamma}^{\infty} g(x) d \beta_{n}(x) .
$$

Using (23), $a_{n}$ and $b_{n}$ can be calculated with $H(\cdot)$ replaced by $\alpha_{n}(\cdot)$ and $\beta_{n}(\cdot)$, respectively. After some lengthy algebraic manipulations, it can be shown that

$$
\begin{aligned}
\alpha_{n}(x)= & \sum_{k=0}^{n-2} \sum_{j=0}^{k} \frac{(\mu(x-z(n-1) \gamma))^{j}}{j !(z+1)^{k-j+1} z^{j-1}} e^{-\mu((1+1 / z) x-n \gamma)} \\
& -e^{\mu(x-\gamma)}
\end{aligned}
$$

and

$$
\beta_{n}(x)=-\frac{\left.e^{-\mu((z+1) x+(z(n-2)-2) \gamma}\right)}{(z+1)^{n-1}} .
$$

From (19)-(29), it can be seen that an exact closed-form expression for the goodput of the MDC system over Rayleigh fading channels has been obtained. 


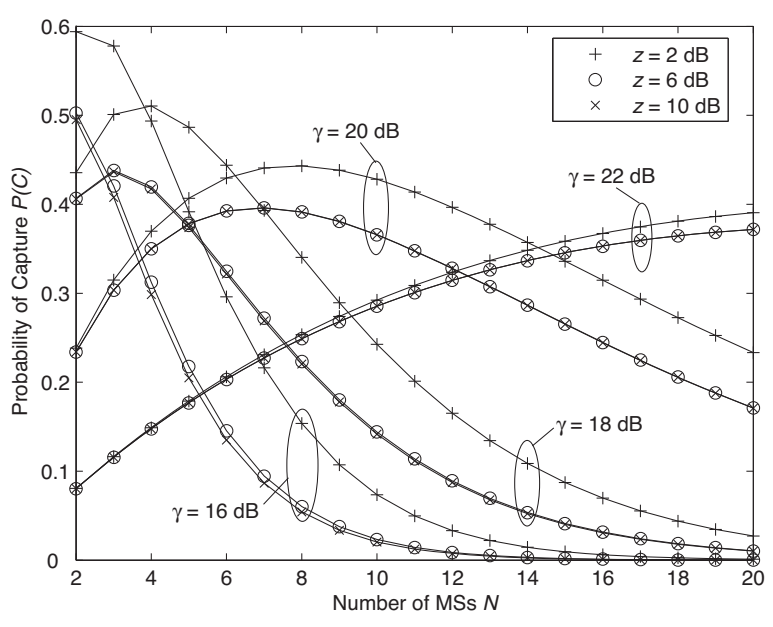

Fig. 2. $P(C)$ over Rayleigh fading channels with an average SNR of 17 dB.

\section{Numerical Results for MDC over Rayleigh Fading Chan- nels}

In this section, we consider the relationship between the expected goodput for MDC over Rayleigh fading channels and the system parameters $N, z$ and $\gamma$. For the numerical results in this section, the average SNR is $17 \mathrm{~dB}$ (i.e., $\mu=0.02$ ) and the rate adaptation policy is the wireless LAN rate adaptation policy as described in Section IV.

We first consider how the performance of MDC is affected by $\gamma$. The increased goodput through exploiting multiuser diversity in MDC comes from the times when the BS is able to capture the best MS. For the MDC protocol, we define the probability of capture $P(C)$ as follows:

$$
P(C)=\sum_{n=1}^{N} P\left(C \mid R_{n}\right) P\left(R_{n}\right) .
$$

Fig. 2 shows the relationship between $P(C)$ and the number of MSs in the network over Rayleigh fading channels. It can be seen from Fig. 2 that as $z$ increases, $P(C)$ decreases. On the other hand, given $N$, there exists an optimal value of $\gamma$ that maximizes the expected goodput for MDC. For example at $N=10$, we find that $P(C)$ first increases as $\gamma$ increases from $16 \mathrm{~dB}$ to $20 \mathrm{~dB}$, but then decreases as $\gamma$ increases from $20 \mathrm{~dB}$ to $22 \mathrm{~dB}$. Further, it can be seen from Fig. 2 that the optimal value of $\gamma$ should increase as $N$ increases. This appeals to intuition, since as the number of MSs increases, we expect that the number of responding MSs to increase. This increase in the number of responding MSs necessitates an increase in $\gamma$ to limit the interference from MSs that are unlikely to have the best channel, so that the BS has the best chance of capturing the best MS.

Fig. 3 shows the expected goodput for MDC and MAD over a Rayleigh fading channel. The numerical results for the goodput performance of MAD is obtained using order statistic analysis. $\gamma$ has been numerically optimized to the nearest 0.1 $\mathrm{dB}$ so that the goodput is maximized for each value of $N$ and $z$. As can be seen from Fig. 3, the numerical results match the simulation results.

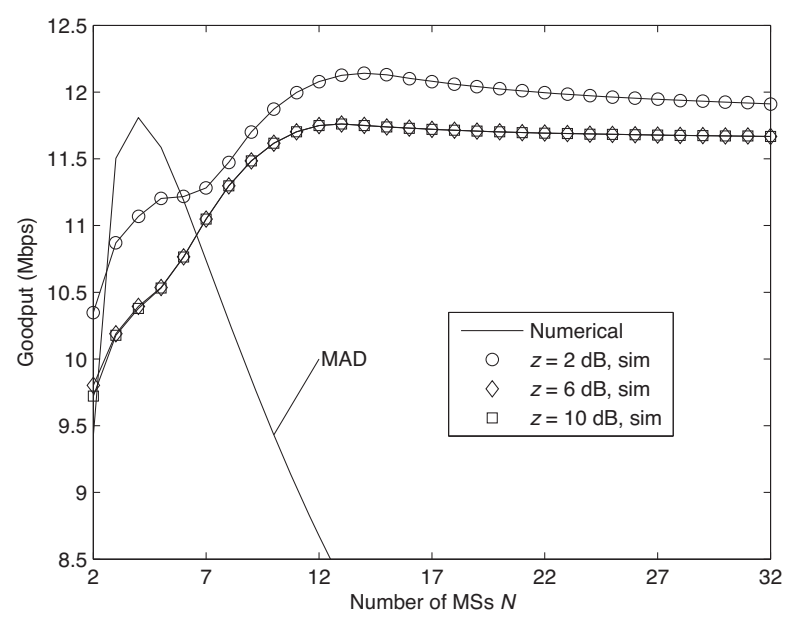

Fig. 3. Expected goodput for MDC over Rayleigh fading channels at an average SNR of $17 \mathrm{~dB}$.

It is apparent from Fig. 3 that MDC achieves better goodput performance than MAD, especially when the capture ratio is small or the number of MSs is reasonably large. The goodput performance of MAD suffers when the number of MSs increases because the overhead incurred from polling all of the MSs in the network outweighs any potential gain from polling more MSs for CSI. It can also be seen that the expected goodput for MDC becomes less sensitive to $z$ as $z$ increases. In particular, there appears to be no difference in goodput performance between $z=6 \mathrm{~dB}$ and $z=10 \mathrm{~dB}$ in Fig. 3 . Hence the goodput plot for $z=6 \mathrm{~dB}$ can be considered to represent a lower bound on the goodput performance of MDC, which is comparable to the performance of MAD in the case where the optimal number of MSs is polled.

\section{An MDC Application Example}

\section{A. Physical Layer and Medium Access Control Layer of IEEE} 802.11a

The PHY of IEEE 802.11a [17] employs Orthogonal Frequency Division Multiplexing (OFDM) technology. There are 52 data subcarriers in each OFDM symbol. Adaptive coding and modulation with eight PHY modes is employed in IEEE 802.11a, which enables rate adaptation. Table I shows the modulation scheme, coding rate (CR), data rate and number of data bits per OFDM symbol ( $\mathrm{N}_{\text {DBPS }}$ ) for each IEEE 802.11a PHY mode.

The IEEE 802.11a PHY is composed of a Physical Medium Dependent (PMD) sublayer and a Physical Layer Convergence Procedure (PLCP) sublayer. The PLCP sublayer provides the interface of services to the medium access control (MAC) layer from the PMD through the service access point (SAP). Wireless stations communicate at the PHY level through PLCP protocol data unit (PPDU) frames. The transmission time of any packet is precisely the transmission time of a PPDU frame in an IEEE 802.11a system.

The PPDU frame format is shown in Fig. 4. It is composed of a training sequence of 10 short symbols and 2 long symbols. The length of the training sequence is $16 \mu \mathrm{s}$. The training 


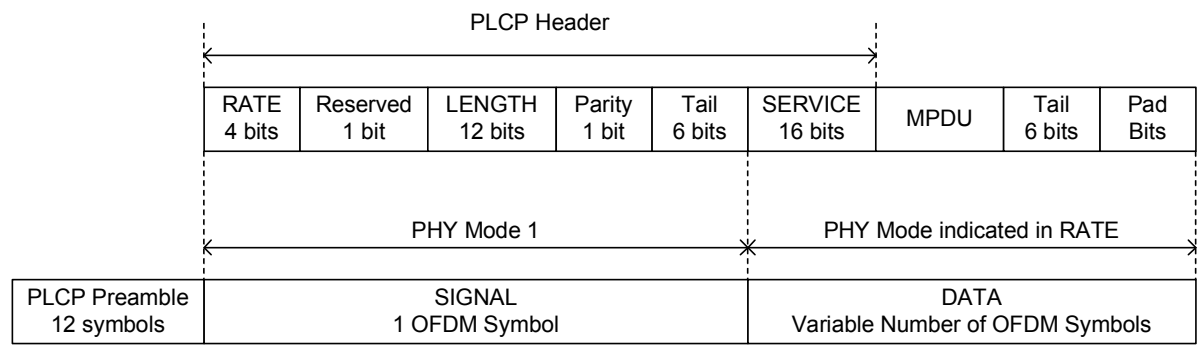

Fig. 4. IEEE 802.11a PPDU frame format.

TABLE I

IEEE 802.11 A PHY MODES

\begin{tabular}{|c|c|c|r|r|}
\hline Mode & Modulation & CR & Data Rate & N $_{\text {DBPS }}$ \\
\hline \hline 1 & BPSK & $1 / 2$ & $6 \mathrm{Mbps}$ & 24 \\
2 & BPSK & $3 / 4$ & $9 \mathrm{Mbps}$ & 36 \\
3 & QPSK & $1 / 2$ & $12 \mathrm{Mbps}$ & 48 \\
4 & QPSK & $3 / 4$ & $18 \mathrm{Mbps}$ & 72 \\
5 & $16-Q A M$ & $1 / 2$ & $24 \mathrm{Mbps}$ & 96 \\
6 & 16-QAM & $3 / 4$ & $36 \mathrm{Mbps}$ & 144 \\
7 & 64-QAM & $2 / 3$ & $48 \mathrm{Mbps}$ & 192 \\
8 & 64-QAM & $3 / 4$ & $54 \mathrm{Mbps}$ & 216 \\
\hline
\end{tabular}

sequence is succeeded by the SIGNAL field, which fits exactly within a PHY mode 1 OFDM symbol. The remaining part of the PPDU frame contains a SERVICE field, the MAC Protocol Data Unit (MPDU), tail bits and pad bits.

The number of data bits per OFDM symbol for each IEEE 802.11a PHY mode is shown in Table II. An OFDM symbol duration is $4 \mu \mathrm{s}$. For a PHY mode $m$ with $b(m)$ data bits per OFDM symbol (i.e., $\mathrm{N}_{\text {DBPS }}$ in Table I), the transmission time $t$ in $\mu$ s of an MPDU frame of size $l$ octets is:

$$
\begin{aligned}
t(m, l) & =16+4+4\left\lceil\frac{16+8 l+6}{b(m)}\right\rceil \\
& =4\left\lceil\frac{8 l+22}{b(m)}+5\right\rceil
\end{aligned}
$$

where $\lceil\cdot\rceil$ is the ceiling function.

\section{B. MDC as Applied in IEEE 802.11a}

In this section, we present an application of MDC using the existing infrastructure from the IEEE 802.11 a system $^{2}$. Fig. 5 shows the IEEE 802.11 [20] MAC frame formats (i.e., the MPDUs) used in the MDC protocol, which include Request to Send (RTS) frames, Clear to Send (CTS) frames, data frames and acknowledgement (ACK) frames. The RTS and CTS frames are short control frames used as part of the optional RTS/CTS mechanism in IEEE 802.11 for channel reservation in its CSMA/CA MAC protocol.

In MDC, $\mathrm{CP}$ and CA packets are implemented as RTS frames, and the PR and CSIF packets are implemented as

\footnotetext{
${ }^{2}$ We wish to modify the IEEE 802.11 a system as little as possible in order to compare the proposed MDC with MAD. In fact, the transmission times of the control packets in MDC can be significantly reduced since the BS only needs to determine the identity of a transmitting MS.
}

TABLE II

MDC DATA TRANSMISSION PARAMETERS

\begin{tabular}{|c||c|c|c|c|c|}
\hline $\begin{array}{c}\text { Data } \\
\text { Mode }\end{array}$ & $\begin{array}{c}\text { Payload } \\
(\text { bytes })\end{array}$ & $\begin{array}{c}t_{\text {data }} \\
(\mu \mathrm{s})\end{array}$ & $\begin{array}{c}\text { ACK } \\
\text { Mode }\end{array}$ & $\begin{array}{c}t_{\text {ACK }} \\
(\mu \mathrm{s})\end{array}$ & $\begin{array}{c}\text { Mode } \\
\text { Threshold }(\mathrm{dB})\end{array}$ \\
\hline \hline 1 & 218 & 336 & 1 & 44 & 9 \\
3 & 485 & 348 & 3 & 32 & 12 \\
4 & 743 & 348 & 3 & 32 & 15 \\
5 & 1013 & 352 & 4 & 28 & 18 \\
6 & 1535 & 352 & 4 & 28 & 21 \\
7 & 2057 & 352 & 4 & 28 & 26 \\
8 & 2304 & 352 & 4 & 28 & 28 \\
\hline
\end{tabular}

CTS frames ${ }^{3}$. RTS and CTS frames are always transmitted using PHY mode 1, hence using (31), we can calculate that their transmission times are $t_{\mathrm{RTS}}=52 \mu \mathrm{s}$ and $t_{\mathrm{CTS}}=44 \mu \mathrm{s}$, respectively.

In the MDC system, PHY mode 2 is not used because the goodput analysis results in [21] have shown that the effective goodput using PHY mode 3 exceeds that of PHY mode 2 at all SNRs for both 200 -byte and 2000-byte payloads. The mode thresholds for each PHY mode have been adjusted upwards by a $5 \mathrm{~dB}$ implementation margin from the theoretical mode thresholds in [21].

According to the IEEE 802.11a standard, all control frames must be transmitted at one of the rates in the mandatory rate set \{6 Mbps, 12 Mbps, 24 Mbps\}. In the MDC system, an ACK packet is transmitted at the highest rate in the mandatory rate set that does not exceed the transmission rate of the associated data packet. Additionally, the largest possible payload of 2304 bytes [21] is transmitted using PHY mode 8 , and the sum of the data packet transmission duration $\left(t_{\text {data }}\right)$ and the ACK packet transmission duration $\left(t_{\mathrm{ACK}}\right)$ is made constant across all PHY modes. Table II shows the payload size, $t_{\text {data }}$, ACK PHY mode, $t_{\mathrm{ACK}}$ and mode threshold for each PHY mode in the MDC system.

Following each step in the MDC transmission cycle, the IEEE 802.11a Short Interframe Space (SIFS), which is $16 \mu \mathrm{s}$ in duration, is used to provide the PHY and MAC the

\footnotetext{
${ }^{3}$ Note that the RTS and CTS frames in IEEE 802.11 should be modified to accommodate the proposed MDC. For the CP packet, the pad bits in the PPDU frame can be used to carry command information. For the PR packet, the Receiver Address field in the CTS frame should be replaced with the Transmitter Address. For the CSIF packet, the Receiver Address field in the CTS frame can be modified to carry CSI.
} 


\begin{tabular}{|c|c|c|c|c|c|c|c|c|c|c|c|}
\hline \multirow{2}{*}{$\begin{array}{l}\text { Octets: } 2 \\
\begin{array}{|l}\text { Frame } \\
\text { Control }\end{array}\end{array}$} & 2 & 6 & 6 & \multicolumn{2}{|l|}{4} & Octets: & \multicolumn{2}{|r|}{2} & \multicolumn{2}{|r|}{6} & \\
\hline & Duration & $\begin{array}{l}\text { Receiver } \\
\text { Address }\end{array}$ & $\begin{array}{l}\text { Transmitter } \\
\text { Address }\end{array}$ & \multicolumn{2}{|c|}{$\begin{array}{c}\text { Frame Check } \\
\text { Sequence }\end{array}$} & \multicolumn{2}{|c|}{$\begin{array}{l}\text { Frame } \\
\text { Control }\end{array}$} & Duration & \multicolumn{2}{|c|}{$\begin{array}{l}\text { Receiver } \\
\text { Address }\end{array}$} & $\begin{array}{r}\text { Frame } \\
\text { Seq }\end{array}$ \\
\hline \multicolumn{7}{|c|}{ (a) RTS frame format } & \multicolumn{5}{|c|}{ (b) CTS frame format } \\
\hline \multirow[t]{2}{*}{ Octets: } & 2 & 2 & 6 & 6 & & 6 & 2 & $0-2$ & & 4 & \\
\hline & $\begin{array}{l}\text { Frame } \\
\text { Control }\end{array}$ & Duration/ID & Address 1 & Address 2 & & dress 3 & $\begin{array}{l}\text { Sequence } \\
\text { Control }\end{array}$ & Frame & & $\begin{array}{l}\text { Frame } \\
\text { Seque }\end{array}$ & $\begin{array}{l}\text { heck } \\
\text { ice }\end{array}$ \\
\hline
\end{tabular}

(c) Data frame format

\begin{tabular}{c|c|c|c|} 
Octets: 2 & 2 & 6 & 4 \\
\hline $\begin{array}{l}\text { Frame } \\
\text { Control }\end{array}$ & Duration & $\begin{array}{c}\text { Receiver } \\
\text { Address }\end{array}$ & $\begin{array}{l}\text { Frame Check } \\
\text { Sequence }\end{array}$ \\
\hline
\end{tabular}

(d) ACK frame format

Fig. 5. IEEE 802.11 MAC frame formats used in MDC.

necessary time to respond to a received transmission ${ }^{4}$. Hence, the resultant MDC transmission cycle duration $t_{\text {cycle }}$ (in $\mu \mathrm{s}$ ) is:

$$
\begin{aligned}
t_{\text {cycle }} & =2 t_{\mathrm{RTS}}+2 t_{\mathrm{CTS}}+t_{\mathrm{data}}+t_{\mathrm{ACK}}+6 \times 16 \\
& =668 .
\end{aligned}
$$

\section{Channel Model}

We consider a wireless channel with path loss, shadowing and multipath fading [22], [23]. We assume that channel gains of different transmission cycles are statistically independent. The instantaneous received power $P_{R}$ of a transmitted radio signal is given by

$$
P_{R}=P_{T} K_{L} K_{S} K_{F}
$$

where $P_{T}$ is the constant network-wide transmission power, $K_{L}$ is the scalar factor that represents path loss, $K_{S}$ is the scalar factor that represents shadowing and $K_{F}$ is the scalar factor that represents multipath fading.

The path loss scalar factor $K_{L}$ is modeled according to the following:

$$
K_{L}(d)=K_{L}\left(d_{0}\right)\left(\frac{d}{d_{0}}\right)^{-\eta} \quad d \geq d_{0}
$$

where $d$ is the transmitter-receiver separation distance, $\eta$ is the path loss exponent and $d_{0}$ is the close-in reference distance. The scalar factor $K_{L}\left(d_{0}\right)$ in (34) is determined from the free space path loss model:

$$
K_{L}\left(d_{0}\right)=\frac{G_{T} G_{R} \lambda^{2}}{\left(4 \pi d_{0}\right)^{2}}
$$

where $G_{T}$ is the transmitter antenna gain, $G_{R}$ is the receiver antenna gain and $\lambda$ is the wavelength of the carrier. We assume that the network covers a circular cell with the BS at its center and MSs are uniformly distributed over a punctured disk with

\footnotetext{
${ }^{4}$ We note that in Step 1 of MDC, the CP packet can carry control information to explicitly enforce that all MSs with a channel state greater than the response threshold to send PR packets after a delay of SIFS duration. As a result, MDC is different from the CSMS/CA mechanism used in IEEE 802.11 systems. On the other hand, at the end of each transmission cycle, the BS may opt to release the channel by not sending the CP packet. In this manner conventional CSMA/CA can be applied in the proposed application example.
}

inner radius $d_{0}$ and outer radius $R$. Hence, the pdf of the distance $d$ between the BS and each MS is

$$
f_{d}(x)=\frac{2 x}{R^{2}-d_{0}^{2}}, \quad d_{0} \leq x \leq R .
$$

The shadowing scalar factor $K_{S}$ is modeled by a log-normal random variable with mean of $0 \mathrm{~dB}$ and a standard deviation of $\sigma_{s} \mathrm{~dB}$. Thus, the pdf of $K_{S}$ is

$$
f_{K_{S}}(x)=\frac{1}{\sigma x \sqrt{2 \pi}} e^{-((\ln x) / \sigma)^{2}}, \quad x>0
$$

where $\sigma=0.1 \sigma_{s} \ln 10$.

Multipath fading is modeled by the Rayleigh fading model and the pdf of the fading scalar factor $K_{F}$ is given by (16) with $\mu=1$. The source of the AWGN wireless channel noise is assumed to be thermal noise and is modeled by

$$
P_{N}=k T B \times 10^{0.1 N F}
$$

where $k$ is the Boltzmann constant, $T$ is the equivalent noise temperature of the wireless environment, $B$ is the bandwidth of the channel and $N F$ is the noise figure of the wireless system.

\section{Simulation Results}

In this section, we present the simulation results using the system described in Section IV. Table III shows the values of the parameters for the wireless channel model used in the simulations. The predicted SNR at the outer radius $R$ due to path loss was chosen to be equal to the mode threshold for PHY mode 3. That is,

$$
10 \log _{10}\left(\frac{P_{T} K_{L}(R)}{P_{N}}\right)=12 \mathrm{~dB} .
$$

Following [24], a standard deviation of $3.8 \mathrm{~dB}$ is used as the shadowing factor $K_{S}$ for the IEEE 802.11a PHY at a carrier frequency of $5.2 \mathrm{GHz}$ in residential environments. The other values in Table III are consistent with those employed in [25].

Fig. 6 shows the effect of the value of $\gamma$ on the goodput performance of MDC for a network with 8 MSs, based the channel model parameters in Table III. It can be seen from Fig. 6 that the optimization of $\gamma$ is important in MDC, as the goodput for MDC is adversely affected by a poor choice of $\gamma$. Further, the optimal value of $\gamma$ increases with both $N$ and 
TABLE III

Values of Wireless Channel Model Parameters

\begin{tabular}{c||c}
\hline Parameter & Value \\
\hline \hline$P_{T}$ & $10 \mathrm{dBm}$ \\
$d_{0}$ & $1 \mathrm{~m}$ \\
$G_{R}$ & $0 \mathrm{dBi}$ \\
$G_{T}$ & $0 \mathrm{dBi}$ \\
$\lambda$ & $0.0577 \mathrm{~m}$ \\
$\eta$ & 3 \\
$R$ & $25.5 \mathrm{~m}$ \\
$\sigma_{s}$ & $3.8 \mathrm{~dB}$ \\
$T$ & $290 \mathrm{~K}$ \\
$B$ & $20 \mathrm{MHz}$ \\
$N F$ & $10 \mathrm{~dB}$ \\
\hline
\end{tabular}

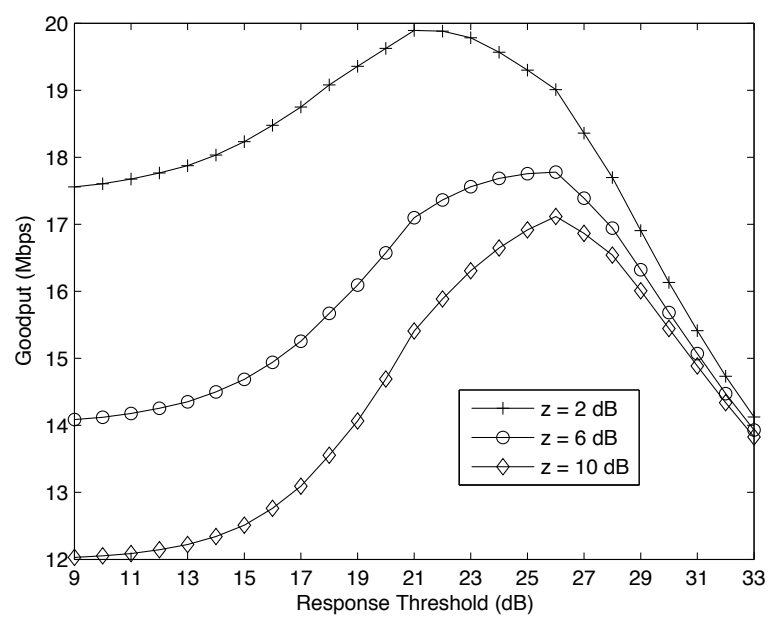

Fig. 6. MDC goodput performance versus $\gamma$ in a network with 8 MSs

$z$. As mentioned in Section III, the optimal value of $\gamma$ should increase as $N$ and/or $z$ increases in order to limit the number of interfering MSs at the channel competition step.

Although it is important to optimize $\gamma$ in MDC, it can be seen from Fig. 6 that the goodput is not sensitive to the value of $\gamma$ when $\gamma$ is near the optimal value. For example, at $z=10$ $\mathrm{dB}$, the optimal value of $\gamma$ is $26 \mathrm{~dB}$, with a goodput of 17.1 Mbps. However, for $\gamma=24 \mathrm{~dB}$, the goodput is $16.7 \mathrm{Mbps}$ and for $\gamma=28 \mathrm{~dB}$, the goodput is $16.5 \mathrm{Mbps}$, which is only a $3 \%$ decrease.

Table IV shows the optimal values of $\gamma$ that maximize the goodput for MDC for the wireless channel described in Table III over various values of $N$ and $z$, obtained through extensive simulations ${ }^{5}$. From Table IV, it can be seen that the optimal

\footnotetext{
${ }^{5}$ In a practical system, the optimal value of $\gamma$ can be found heuristically using iterative optimization algorithms. For a given value of $\gamma$, the BS can estimate the expected goodput, $E[G]$, by observing the average goodput over a number of transmission cycles. Since $E[G]$ is a continuous function of $\gamma$ (e.g., see Fig. 6), the BS can find the optimal value of $\gamma$ heuristically using the estimates of $E[G]$ for different values of $\gamma$. Alternatively, the BS can observe the probability of capture, $P(C)$, over a number of transmission cycles and adjust $\gamma$ to maximize $P(C)$. In our ongoing work, we have found that over Rayleigh fading channels, the value of $\gamma$ that optimizes $P(C)$ is very close to the value of $\gamma$ that optimizes $E[G]$.
}

TABLE IV

OPTIMAL RESPONSE THRESHOLDS $\gamma$ FOR MDC BASED ON THE WIRELESS CHANNEL IN TABLE III

\begin{tabular}{|r||r|r|r|}
\hline$N$ & $z=2 \mathrm{~dB}$ & $z=6 \mathrm{~dB}$ & $z=10 \mathrm{~dB}$ \\
\hline \hline 2 & $9 \mathrm{~dB}$ & $12 \mathrm{~dB}$ & $15 \mathrm{~dB}$ \\
3 & $15 \mathrm{~dB}$ & $18 \mathrm{~dB}$ & $21 \mathrm{~dB}$ \\
4 & $17 \mathrm{~dB}$ & $21 \mathrm{~dB}$ & $21 \mathrm{~dB}$ \\
5 & $18 \mathrm{~dB}$ & $21 \mathrm{~dB}$ & $22 \mathrm{~dB}$ \\
6 & $21 \mathrm{~dB}$ & $21 \mathrm{~dB}$ & $24 \mathrm{~dB}$ \\
7 & $21 \mathrm{~dB}$ & $24 \mathrm{~dB}$ & $26 \mathrm{~dB}$ \\
8 & $21 \mathrm{~dB}$ & $26 \mathrm{~dB}$ & $26 \mathrm{~dB}$ \\
9 & $22 \mathrm{~dB}$ & $26 \mathrm{~dB}$ & $26 \mathrm{~dB}$ \\
10 & $23 \mathrm{~dB}$ & $26 \mathrm{~dB}$ & $26 \mathrm{~dB}$ \\
11 & $24 \mathrm{~dB}$ & $26 \mathrm{~dB}$ & $27 \mathrm{~dB}$ \\
12 & $26 \mathrm{~dB}$ & $27 \mathrm{~dB}$ & $28 \mathrm{~dB}$ \\
13 & $26 \mathrm{~dB}$ & $27 \mathrm{~dB}$ & $28 \mathrm{~dB}$ \\
14 & $26 \mathrm{~dB}$ & $28 \mathrm{~dB}$ & $28 \mathrm{~dB}$ \\
15 & $26 \mathrm{~dB}$ & $28 \mathrm{~dB}$ & $28 \mathrm{~dB}$ \\
16 & $26 \mathrm{~dB}$ & $28 \mathrm{~dB}$ & $29 \mathrm{~dB}$ \\
\hline
\end{tabular}

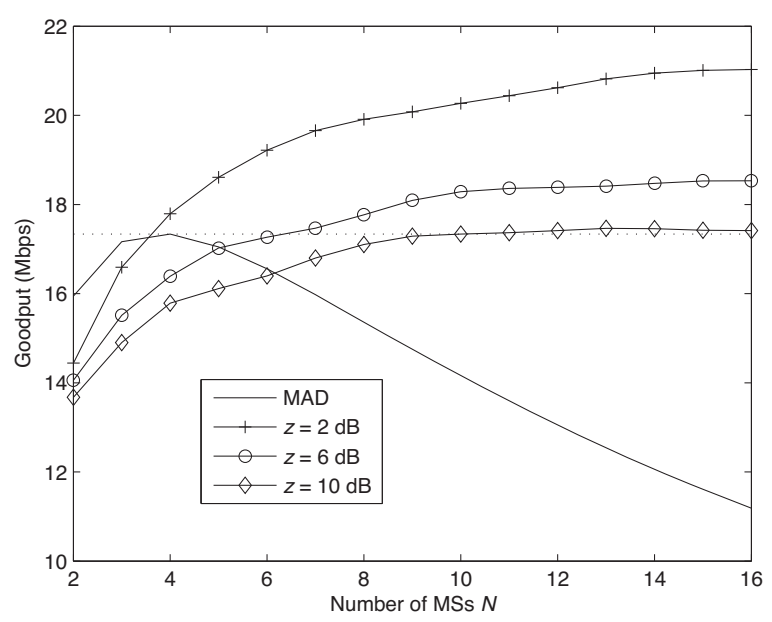

Fig. 7. MAD goodput performance and MDC goodput performance with optimal response thresholds

value of $\gamma$ is a non-decreasing function of $N$ and $z$. This can be explained from the perspective of the probability of capture, $P(C)$. When the number of competing MSs is large, increasing $\gamma$ reduces the number of competing MSs during channel competition, thereby increasing $P(C)$. However, the $P(C)$ decreases with the increase of $N$, due to the increased number of competing MSs during the channel competition step. Likewise, the $P(C)$ decreases as $z$ increases, as the channel state of the strongest PR signal must be higher.

Fig. 7 shows the expected goodput performance for MDC using the optimal response thresholds from Table IV. The expected goodput for MAD when all of the MSs in the network are polled is also shown in Fig. 7. In simulating the MAD protocol, we have used the CTS and ACK packet formats described in the IEEE 802.11 standard rather than the modified versions described in [7], since our channel model assumes channel reciprocity and we have not used packet 
concatenation $^{6}$. Unlike MDC, a broadcast packet is insufficient for the channel probing step in MAD because the MSs must know in which order the reply messages are to be sent. Therefore, the format of the Group Request to Send (GRTS) frame in [7] for MAD has been maintained. As in MDC, the total duration for data transmission and acknowledgement is maintained constant for MAD, based on the data transmission parameters from Table II. The SIFS is also used between each MAD packet transmission. Thus, the cycle time for MAD is $(8 N+532) \mu \mathrm{s}$.

From Fig. 7, it can be seen that by employing the capture effect, MDC enjoys reduced overhead while exploiting multiuser diversity. Since the overhead in MDC is constant and independent of $N$, we find that the expected goodput for MDC increases with $N$ in Fig. 7. On the other hand, the overhead for MAD outweighs the multiuser diversity gain. These trends are similar to what we have observed in the numerical results for Rayleigh fading channels in Section III.

The expected MDC goodput curves for $z=2 \mathrm{~dB}$ and $z=6$ $\mathrm{dB}$ show an appreciable performance improvement over the optimal MAD goodput. Even for $z=10 \mathrm{~dB}$, the expected goodput for MDC is higher than the optimal MAD goodput when $N$ is large enough. It is particularly noteworthy that the expected goodput for MDC becomes less sensitive to $z$ as $z$ increases.

\section{CONCLUSION}

In this paper, we have proposed a new wireless MAC protocol called MDC that explicitly employs the capture effect to find the MS with the best channel in a network with many MSs. Our approach presents an effective solution to the overhead problem in a network exploiting multiuser diversity, due to the requirement for CSI feedback. We have developed a general analytical framework for determining the goodput of MDC, which we have verified with simulations on Rayleigh fading channels. We have also investigated the performance of MDC as applied in IEEE 802.11a systems under a wireless environment with path loss, shadowing and Rayleigh fading. Based on the simulation results, we have observed that when the number of MSs in the network is sufficiently large, MDC is able to exploit multiuser diversity more effectively than the MAD scheme in the literature.

\section{APPENDIX}

$$
\begin{gathered}
\text { DERIVATION OF } P\left(R_{n}\right), P\left(C \mid R_{n}\right), P\left(\bar{C} \mid R_{n}\right), E\left[G \mid C, R_{n}\right] \\
\text { AND } E\left[G \mid \bar{C}, R_{n}\right]
\end{gathered}
$$

For each transmission cycle, the probability that MS $i$ sends a PR packet is $P\left(\Gamma_{i}>\gamma\right)=1-F_{\Gamma}(\gamma)$. Since the $\Gamma_{i}$ s are i.i.d, the probability that exactly $n$ MSs send a PR packet to the $\mathrm{BS}$ is given by the binomial distribution:

$$
P\left(R_{n}\right)=\left(\begin{array}{c}
N \\
n
\end{array}\right)\left(1-F_{\Gamma}(\gamma)\right)^{n} F_{\Gamma}(\gamma)^{N-n} .
$$

${ }^{6}$ The modified CTS packet in [7] contains an extra field that carries CSI and the modified ACK packet in [7] contains an extra field that enables acknowledgement of multiple packets within a superframe.
$P\left(C \mid R_{n}\right)$ is the probability that the BS is able to correctly identify the MS with the best channel at the channel competition step given that $n$ MSs send a PR packet. From the probability axioms, we have that for all $1 \leq n \leq N$,

$$
P\left(\bar{C} \mid R_{n}\right)=1-P\left(C \mid R_{n}\right) .
$$

Since $C \cap R_{0}=\emptyset$ and $C \cap R_{1}=R_{1}$,

$$
P\left(C \mid R_{0}\right)=0
$$

and

$$
P\left(C \mid R_{1}\right)=1
$$

For $n \geq 2$, the event $C \cap R_{n}$ occurs when exactly $n$ MSs send a PR packet to the BS and the SNR of the winning MS (i.e., MS $w$ ) exceeds $z$ times the sum of the SNRs of the other $(n-1)$ replying MSs. Define the event $E_{k, n}$ as

$$
E_{k, n}=\left\{\Gamma_{v_{k}}>z \sum_{i=1, i \neq k}^{n} \Gamma_{v_{i}}\right\}
$$

The event $E_{k, n}$ implies that MS $v_{k}$ will be captured at the BS given that $n$ MSs send a PR packet. Since $\Gamma_{v_{k}} \geq 0$ for $1 \leq k \leq n$ and we assume that $z \geq 1$, it follows that $E_{1, n}, E_{2, n}, \ldots, E_{n, n}$ are mutually exclusive. Further, since the $\Gamma_{v_{k}}$ s are i.i.d., $P\left(E_{k, n}\right)$ is independent of $k$. In other words, only one MS can be captured at the BS at the channel competition step and each MS has an equal probability of being captured. Thus, for $n \geq 2$,

$$
\begin{aligned}
P\left(C \mid R_{n}\right) & =P\left(\bigcup_{k=1}^{n} E_{k, n}\right) \\
& =\sum_{k=1}^{n} P\left(E_{k, n}\right) \\
& =n P\left(E_{1, n}\right) \\
& =n P\left(\Gamma_{v_{1}}>z \sum_{i=2}^{n} \Gamma_{v_{i}}\right) \\
& =n \int_{(n-1) \gamma}^{\infty} \int_{z x}^{\infty} f_{V}(y) f_{V, n-1}(x) d y d x \\
& =n \int_{(n-1) \gamma}^{\infty}\left(1-F_{V}(z x)\right) f_{V, n-1}(x) d x .
\end{aligned}
$$

To derive $E\left[G \mid C, R_{n}\right]$ and $E\left[G \mid \bar{C}, R_{n}\right]$, we first note that $G=g\left(\Gamma_{w}\right)$. It directly follows that

$$
\begin{gathered}
E[G]=\int_{0}^{\infty} g(x) d P\left(\Gamma_{w} \leq x\right), \\
E\left[G \mid C, R_{n}\right]=\int_{0}^{\infty} g(x) d P\left(\Gamma_{w} \leq x \mid C, R_{n}\right)
\end{gathered}
$$

and

$$
E\left[G \mid \bar{C}, R_{n}\right]=\int_{0}^{\infty} g(x) d P\left(\Gamma_{w} \leq x \mid \bar{C}, R_{n}\right) .
$$

Since $C \cap R_{0}=\bar{C} \cap R_{1}=\emptyset$, we define

$$
E\left[G \mid C, R_{0}\right]=0
$$

and

$$
E\left[G \mid \bar{C}, R_{1}\right]=0 .
$$


Note that $\bar{C} \cap R_{0}=R_{0}$. This implies that the set of $\Gamma_{i} \mathrm{~s}$ is equal to the set of $\Gamma_{u_{j}}$ s. Then,

$$
\begin{aligned}
& P\left(\Gamma_{w} \leq x \mid \bar{C}, R_{0}\right) \\
= & \sum_{i=1}^{N} P\left(\Gamma_{w} \leq x \mid \bar{C}, R_{0}, w=i\right) P\left(w=i \mid \bar{C}, R_{0}\right) \\
= & \sum_{j=1}^{N} P\left(\Gamma_{w} \leq x \mid w=u_{j}\right) P\left(w=u_{j}\right) \\
= & P\left(\Gamma_{u_{1}} \leq x\right) \\
= & F_{U}(x)
\end{aligned}
$$

where in the third step, we have used the fact that $P(w=$ $\left.u_{j}\right)=1 / N$ and $P\left(\Gamma_{u_{j}} \leq x\right)$ is independent of $j$. Using (48), we have

$$
E\left[G \mid \bar{C}, R_{0}\right]=\int_{0}^{\gamma} g(x) f_{U}(x) d x .
$$

Similarly, the event $C \cap R_{1}=R_{1}$ means that the winner is the only replying MS (i.e. $\Gamma_{w}=\Gamma_{v_{1}}$ ). Hence,

$$
\begin{aligned}
P\left(\Gamma_{w} \leq x \mid C, R_{1}\right) & =P\left(\Gamma_{v_{1}} \leq x\right) \\
& =F_{V}(x)
\end{aligned}
$$

and thus from (47)

$$
E\left[G \mid C, R_{1}\right]=\int_{\gamma}^{\infty} g(x) f_{V}(x) d x .
$$

In the following, we derive $P\left(\Gamma_{w} \leq x \mid C, R_{n}\right)$ for $n \geq 2$. Since the $\Gamma_{v_{k}}$ s are i.i.d., it follows that

$$
\begin{aligned}
& P\left(\Gamma_{w} \leq x \mid C, R_{n}\right) \\
= & \sum_{k=1}^{n} P\left(\Gamma_{w} \leq x \mid C, R_{n}, w=v_{k}\right) P\left(w=v_{k} \mid C, R_{n}\right) \\
= & P\left(\Gamma_{w} \leq x \mid C, R_{n}, w=v_{1}\right) \\
= & P\left(\Gamma_{v_{1}} \leq x \mid E_{1, n}\right) \\
= & \frac{P\left(\Gamma_{v_{1}} \leq x, E_{1, n}\right)}{P\left(E_{1, n}\right)} \\
= & \frac{1}{P\left(E_{1, n}\right)} P\left(z \sum_{i=2}^{n} \Gamma_{v_{i}}<\Gamma_{v_{1}} \leq x\right) \\
= & \frac{n}{P\left(C \mid R_{n}\right)} \int_{z(n-1) \gamma}^{x} \int_{(n-1) \gamma}^{y / z} f_{V, n-1}(u) f_{V}(y) d u d y \\
= & \frac{n}{P\left(C \mid R_{n}\right)} \int_{z(n-1) \gamma}^{x} F_{V, n-1}\left(\frac{y}{z}\right) f_{V}(y) d y
\end{aligned}
$$

where in the second step, we have used the fact that $P(w=$ $\left.v_{k} \mid C, R_{n}\right)=1 / n$ and $P\left(\Gamma_{v_{k}} \leq x \mid C, R_{n}\right)$ is independent of $k$.

Using (47), we have that for $n \geq 2$,

$E\left[G \mid C, R_{n}\right]=\frac{n}{P\left(C \mid R_{n}\right)} \int_{z(n-1) \gamma}^{\infty} g(x) F_{V, n-1}\left(\frac{x}{z}\right) f_{V}(x) d x$.

Therefore, from (49), (54) and (56), $E\left[G \mid C, R_{n}\right]$ is given by (11) with $a_{n}$ given by (12).

In the following, we derive $P\left(\Gamma_{w} \leq x \mid \bar{C}, R_{n}\right)$ for $n \geq 2$, so that $E\left[G \mid \bar{C}, R_{n}\right]$ can be calculated using (48). When $n$ MSs send a PR packet simultaneously and the BS is unable to identify the MS with the best channel, the BS will allocate the channel to a randomly chosen MS. The index of the randomly chosen MS is either in $\left\{u_{i}\right\}$ or $\left\{v_{i}\right\}$. Thus,

$$
P\left(w=u_{i} \mid \bar{C}, R_{n}\right)=\frac{1}{N}, \quad 1 \leq i \leq N-n
$$

and

$$
P\left(w=v_{j} \mid \bar{C}, R_{n}\right)=\frac{1}{N}, \quad 1 \leq j \leq n .
$$

Since the $\Gamma_{u_{i}} \mathrm{~s}$ are i.i.d and the $\Gamma_{v_{j}} \mathrm{~s}$ are also i.i.d,

$$
\begin{aligned}
& P\left(\Gamma_{w} \leq x \mid \bar{C}, R_{n}\right) \\
= & \sum_{i=1}^{N-n} P\left(\Gamma_{w} \leq x \mid \bar{C}, R_{n}, w=u_{i}\right) P\left(w=u_{i} \mid \bar{C}, R_{n}\right) \\
& +\sum_{j=1}^{n} P\left(\Gamma_{w} \leq x \mid \bar{C}, R_{n}, w=v_{j}\right) P\left(w=v_{j} \mid \bar{C}, R_{n}\right) \\
= & \frac{N-n}{N} P\left(\Gamma_{u_{1}} \leq x \mid \bar{C}, R_{n}\right)+\frac{n}{N} P\left(\Gamma_{v_{1}} \leq x \mid \bar{C}, R_{n}\right) \\
= & \frac{N-n}{N} F_{U}(x)+\frac{n}{N} P\left(\Gamma_{v_{1}} \leq x \mid \bar{C}, R_{n}\right) .
\end{aligned}
$$

$P\left(\Gamma_{v_{1}} \leq x \mid \bar{C}, R_{n}\right)$ is the conditional cdf of $\Gamma_{v_{1}}$ given that $n$ MSs send a PR packet and the BS is unable to capture the strongest signal. Hence for $n \geq 2$,

$$
\begin{aligned}
& P\left(\Gamma_{v_{1}} \leq x \mid \bar{C}, R_{n}\right) \\
= & \frac{P\left(\Gamma_{v_{1}} \leq x, \bar{C} \mid R_{n}\right)}{P\left(\bar{C} \mid R_{n}\right)} \\
= & \frac{1}{P\left(\bar{C} \mid R_{n}\right)} P\left(\Gamma_{v_{1}} \leq x, \bigcap_{k=1}^{n} \bar{E}_{k, n}\right) \\
= & \frac{1}{P\left(\bar{C} \mid R_{n}\right)}\left(P\left(\Gamma_{v_{1}} \leq x\right)-P\left(\Gamma_{v_{1}} \leq x, \bigcup_{k=1}^{n} E_{k, n}\right)\right) \\
= & \frac{1}{P\left(\bar{C} \mid R_{n}\right)}\left(P\left(\Gamma_{v_{1}} \leq x\right)-\sum_{k=1}^{n} P\left(\Gamma_{v_{1}} \leq x, E_{k, n}\right)\right)
\end{aligned}
$$

where we have used de Morgan's law in the third step and the fact that the $E_{k, n} \mathrm{~s}$ are mutually exclusive in the final step.

Now consider $P\left(\Gamma_{v_{1}} \leq x, E_{k, n}\right)$. For $k=1$, we know from the derivation of $P\left(\Gamma_{w} \leq x \mid C, R_{n}\right)$ that

$$
P\left(\Gamma_{v_{1}} \leq x, E_{1, n}\right)=\int_{z(n-1) \gamma}^{x} F_{V, n-1}(y / z) f_{V}(y) d y .
$$

Since the $\Gamma_{v_{k}}$ s are i.i.d., we have that for $k>1, P\left(\Gamma_{v_{1}} \leq\right.$ $\left.x, E_{k, n}\right)=P\left(\Gamma_{v_{1}} \leq x, E_{2, n}\right)$. Hence for $n \geq 2$,

$$
\begin{aligned}
& \sum_{k=1}^{n} P\left(\Gamma_{v_{1}} \leq x, E_{k, n}\right) \\
= & (n-1) P\left(\Gamma_{v_{1}} \leq x, E_{2, n}\right) \\
= & (n-1) P\left(\Gamma_{v_{1}} \leq x, \Gamma_{v_{2}}>z \sum_{i=1, i \neq 2}^{n} \Gamma_{v_{i}}\right) .
\end{aligned}
$$


For $n>2$ in (62), we have

$$
\begin{aligned}
& P\left(\Gamma_{v_{1}} \leq x, E_{2, n}\right) \\
= & P\left(\Gamma_{v_{1}} \leq x, \Gamma_{v_{2}}>z\left(\Gamma_{v_{1}}+\sum_{i=3}^{n} \Gamma_{v_{i}}\right)\right) \\
= & \int_{\gamma}^{x} \int_{(n-2) \gamma}^{\infty} \int_{z(y+u)}^{\infty} f_{V}(t) f_{V, n-2}(u) f_{V}(y) d t d u d y \\
= & \int_{\gamma}^{x} \int_{(n-2) \gamma}^{\infty}\left(1-F_{V}(z(y+u))\right) f_{V, n-2}(u) f_{V}(y) d u d y .
\end{aligned}
$$

For $n=2$ in (62), we have

$$
\begin{aligned}
P\left(\Gamma_{v_{1}} \leq x, E_{2,2}\right) & =P\left(\Gamma_{v_{1}} \leq x, \Gamma_{v_{2}}>z \Gamma_{v_{1}}\right) \\
& =\int_{\gamma}^{x} \int_{z y}^{\infty} f_{V}(u) f_{V}(y) d u d y \\
& =\int_{\gamma}^{x}\left(1-F_{V}(z y)\right) f_{V}(y) d y .
\end{aligned}
$$

Substituting $n=2$ in (63), we can observe from the sifting property of the Dirac delta function that (64) is actually a special case of (63).

Therefore, putting (59)-(63) into (48), it can be shown that for $n \geq 2$,

$$
\begin{aligned}
E\left[G \mid \bar{C}, R_{n}\right] & =\int_{0}^{\infty} g(x) d P\left(\Gamma_{w} \leq x \mid \bar{C}, R_{n}\right) \\
& =\frac{N-n}{N} E\left[G \mid \bar{C}, R_{0}\right]+\frac{n}{N P\left(\bar{C} \mid R_{n}\right)} b_{n}
\end{aligned}
$$

where $b_{n}$ is given by (14). Thus, from (50), (52) and (65), $E\left[G \mid \bar{C}, R_{n}\right]$ is given by (11) with $a_{n}, b_{n}$ and $c_{n}$ given by (12), (14) and (15), respectively.

\section{REFERENCES}

[1] R. Knopp and P. A. Humblet, "Information capacity and power control in single-cell multiuser communications," in Proc. IEEE ICC'95, vol. 1, Seattle, Jun. 1995, pp. 331-335.

[2] X. Qin and R. Berry, "Exploiting multiuser diversity for medium access control in wireless networks," in IEEE INFOCOM'03, vol. 2, Mar./Apr. 2003, pp. 1084-1094.

[3] P. Bender, P. Black, M. Grob, R. Padovani, N. Sindhashayana, and A. Viterbi, "CDMA/HDR: A bandwidth-efficient high-speed wireless data service for nomadic users," IEEE Commun. Mag., vol. 38, no. 7, pp. 70-77, Jul. 2000.

[4] S. Parkvall, E. Englund, M. Lundevall, and J. Torsner, "Evolving 3G mobile systems: Broadband and broadcast services in WCDMA," IEEE Commun. Mag., vol. 44, no. 2, pp. 30-36, Feb. 2006.

[5] D. Huang and Y. J. Zhang, "User identification for opportunistic OFDM based broadband wireless network," in Proc. IEEE WCNC'07, Mar. 2007, pp. 373-377.

[6] D. Gesbert and M.-S. Alouini, "How much feedback is multi-user diversity really worth?" in Proc. IEEE ICC'04, vol. 1, Jun. 2004, pp. 234-238.

[7] Z. Ji, Y. Yang, J. Zhou, M. Takai, and R. Bagrodia, "Exploiting medium access diversity in rate adaptive wireless LANs," in Proc MOBICOM'04, Sep./Oct. 2004, pp. 345-359.

[8] A. Kamerman and L. Monteban, "WaveLAN II: A high-performance wireless LAN for the unlicensed band," Bell Labs Technical Journal, vol. 2, no. 3, pp. 118-133, 1997.

[9] B. Sadeghi, V. Kanodia, A. Sabharwal, and E. Knightly, "OAR: An opportunistic auto-rate media access control protocol for ad hoc networks," in Proc. MOBICOM'02, Atlanta, Sep. 2002, pp. 24-35.

[10] J. C. Arnbak and W. van Blitterswijk, "Capacity of slotted ALOHA in Rayleigh-fading channels," IEEE J. Select. Areas Commun., vol. 5, no. 2, pp. 216-269, Feb. 1987.
[11] B. Ramamurthi, A. A. Saleh, and D. J. Goodman, "Perfect-capture ALOHA for local radio communications," IEEE J. Select. Areas Commun., vol. 5, no. 5, pp. 806-814, Jun. 1987.

[12] C. Namislo, "Analysis of mobile radio slotted ALOHA networks," IEEE J. Select. Areas Commun., vol. 2, no. 4, pp. 199-204, Jul. 1984.

[13] M. Zorzi and R. R. Rao, "Capture and retransmission control in mobile radio," IEEE J. Select. Areas Commun., vol. 12, no. 8, pp. 1289-1298, Oct. 1994.

[14] J. H. Kim and J. K. Lee, "Capture effects of wireless CSMA/CA protocols in rayleigh and shadow fading channels," IEEE Trans. Veh. Technol., vol. 48, no. 4, pp. 1277-1286, Jul. 1999.

[15] N. Zhang, B. Vojcic, M. R. Souryal, and E. G. Larsson, "Exploiting multiuser diversity in random reservation access," IEEE Trans. Wireless Commun., vol. 5, no. 9, pp. 2548-2554, Sep. 2006.

[16] V. Lau, Y. Liu, and T.-A. Chen, "On the design of mimo block-fading channels with feedback-link capacity constraint," IEEE Trans. Commun., vol. 52, no. 1, pp. 62-70, Jan. 2004.

[17] Wireless LAN Medium Access Control (MAC) and Physical Layer (PHY) Specifications: High Speed Physical Layer in the $5 \mathrm{GHz}$ Band, IEEE Std. 802.11a, Sep. 1999.

[18] C. T. Lau and C. Leung, "Capture models for mobile packet radio networks," IEEE Trans. Commun., vol. 40, no. 1, pp. 917-925, May 1992.

[19] J.-P. M. Linnartz, R. Hekmat, and R.-J. Venema, "Near-far effects in land mobile random access networks with narrow-band Rayleigh fading channels," IEEE Trans. Veh. Technol., vol. 41, no. 1, pp. 77-90, Feb. 1992.

[20] Wireless LAN Medium Access Control (MAC) and Physical Layer (PHY) Specifications, IEEE Std. 802.11, Aug. 1999.

[21] D. Qiao, S. Choi, and K. Shin, "Goodput analysis and link adaptation for IEEE 802.11a wireless LANs," IEEE Trans. Mobile Comput., vol. 1, no. 4, pp. 278-292, Oct./Dec. 2002.

[22] Y. Xue and T. Kaiser, "Pursuing multiuser diversity in an OFDM system with decentralized channel state information," in Proc. IEEE ICC'04, vol. 6, Jun. 2004, pp. 3299-3303.

[23] B. Sklar, "Rayleigh fading channels in mobile digital communication systems part 1: Characterization," IEEE Commun. Mag., vol. 35, no. 9, pp. 136-146, Jul. 1997.

[24] J. Bellorado, S. Ghassemzadeh, L. Greenstein, T. Sveinsson, and V. Tarokh, "Coexistence of ultra-wideband systems with IEEE-802.11a wireless LANs," in IEEE GLOBECOM'03, vol. 1, Dec. 2003, pp. 410414.

[25] O. Awoniyi and F. A. Tobagi, "Effect of fading on the performance of VoIP in IEEE 802.11a WLANs," in IEEE ICC'04, vol. 6, Jun. 2004, pp. 3712-3717.

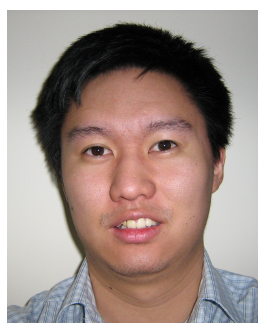

Justin Foo received his B.E. degree in information technology from the University of Western Australia in 2003. He is currently pursuing his Ph.D. degree in information technology at the University of Western Australia. His research interests include packet scheduling, wireless MAC protocols, multiuser diversity and cross-layer design.

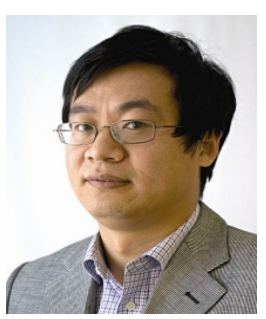

Defeng (David) Huang (M'01 - S'02 - M'05 SM'07) received the B. E. E. E. and M. E. E. E. degree in electronic engineering from Tsinghua University, Beijing, China, in 1996 and 1999, respectively, and the Ph.D. degree in electrical and electronic engineering from the Hong Kong University of Science and Technology (HKUST), Kowloon, Hong Kong, in 2004.

Currently, he is a senior lecturer with School of Electrical, Electronic and Computer Engineering at the University of Western Australia. Dr. Huang serves as an Editor for the IEEE Transactions on Wireless Communications. 